\title{
Active extraperitoneal hematoma in the anterior abdominal wall after blunt injury
}

\author{
Hancheol Jo, Jeongseok Yun, Dong Hun Kim \\ Department of Trauma Surgery, Trauma Center, Dankook University Hospital, Cheonan, \\ Korea
}

Received August 16, 2021

Revised September 5, 2021

Accepted October 15, 2021

Correspondence to

Dong Hun Kim

Department of Trauma Surgery, Trauma

Center, Dankook University Hospital,

201 Manghyang-ro, Dongnam-gu,

Cheonan 31116, Korea

Tel: +82-41-550-3042

Fax: +82-41-550-0039

E-mail: saint7331@gmail.com
A 65-year-old male patient had extraperitoneal hematoma of the anterior abdominal wall after blunt trauma. The patient was treated with conservative management because of stable hemodynamics without deterioration of physical examination and laboratory tests; however, computed tomography revealed active bleeding. In abdominal wall hematoma, physical examination, hemodynamic monitoring, and laboratory tests may be the most important factor in determining a therapeutic plan. Repetitive imaging can be obtained and invasive treatment should be considered if these indices suggest hematoma exacerbation. Patients with abdominal trauma who have altered coagulation status should be carefully monitored for abdominal wall hematoma. Detecting abdominal wall hematoma is important because it may be the clue for potential intra-abdominal organ injury or delayed complication.

Key Words: Abdominal wall, Hematoma, Blunt trauma, Rectus abdominis

\section{Introduction}

Abdominal wall hematoma is a relatively uncommon diagnosis in patients with blunt abdominal trauma. The most common risk factor of abdominal wall hematoma is coagulopathy (1). It is usually self-limiting and treated with conservative management in stable patients whereas expanding hematoma in hemodynamically unstable patients may need surgical or angiographic treatment (2). The conservative management for an extraperitoneal hematoma on the anterior midline abdominal wall following blunt trauma in a patient who was treated successfully with conservative management was described in this study.

\section{Case presentation}

A 65-year-old man with abdominal pain was referred to the emergency department from an outside clinic after blunt trauma with a fall injury. The patient's epigastric area collided with a prominent structure when the patient fell from a 1-m forklift. Moreover, the patient had a medical history of hypertension and hyperlipidemia under medication, without any anticoagulation drug. Upon admission, the patient was alert with a blood pressure of 172/100 $\mathrm{mmHg}$, pulse rate of 62 beats per min, and hemoglobin level of $15.2 \mathrm{~g} / \mathrm{dL}$. Prehospital time was $3.35 \mathrm{~h}$. The patient's abdomen was soft, flat, and nontender on physical examination.

(c)This is an Open Access article distributed under the terms of the Creative Commons Attribution Non-Commercial License (http://creativecommons.org/licenses/by-nc/4.0) which permits unrestricted noncommercial use, distribution, and reproduction in any medium, provided the original work is properly cited.

Copyright (C) 2021 Korean Association for Research, Procedures and Education on Trauma. All rights reserved. 

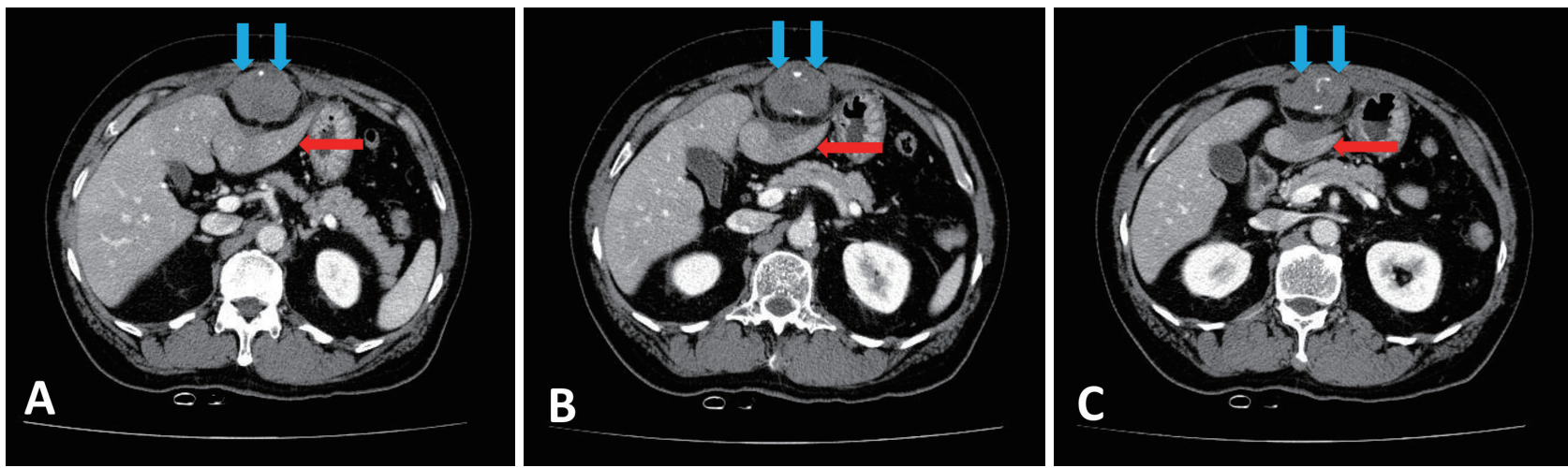

Fig. 1. The computed tomography shows extraperitoneal hematoma and extravasation (blue arrows). The hematoma presses the left lateral segment of liver posteriorly (red arrow) and extends up to umbilical fissure along falciform ligament (A, B, C).

The focused assessment with sonography showed no fluid collection in the abdomen and trauma series radiographs presented no specific injury. However, abdominal computed tomography (CT) revealed an extraperitoneal hematoma with active bleeding in the midline anterior abdominal wall, extending along the falciform ligament (Figs. 1 and 2). No intraperitoneal organ injury was otherwise reported. On repeated physical examination, the patient kept hemodynamically stable and presented no peritoneal irritation sign; however, contrast extravasation was noted in the CT image.

The patient's hemodynamics was stable and hemoglobin level did not decrease throughout the hospital course. The patient was treated with conservative management without transfusion. The patient was allowed to take sips of water and dietary intake on hospital days 2 and 3, respectively. The patient was discharged on hospital day 5 .

\section{Discussion}

The extraperitoneal hematoma of this patient was in the midline abdominal wall and extends along the falciform ligament, which suggested that abdominal trauma caused the injury in the patient's umbilical vessel. The patient was successfully treated with conservative management; however, the CT images showed active bleeding. Many studies report rectus sheath hematoma (RSH) after abdominal blunt trauma, whereas few studies describe traumatic umbilical vessel injury.

Detecting abdominal wall injuries is important because their detection can lead to the clinically important diagnosis of other potential injuries. Missing these injuries may

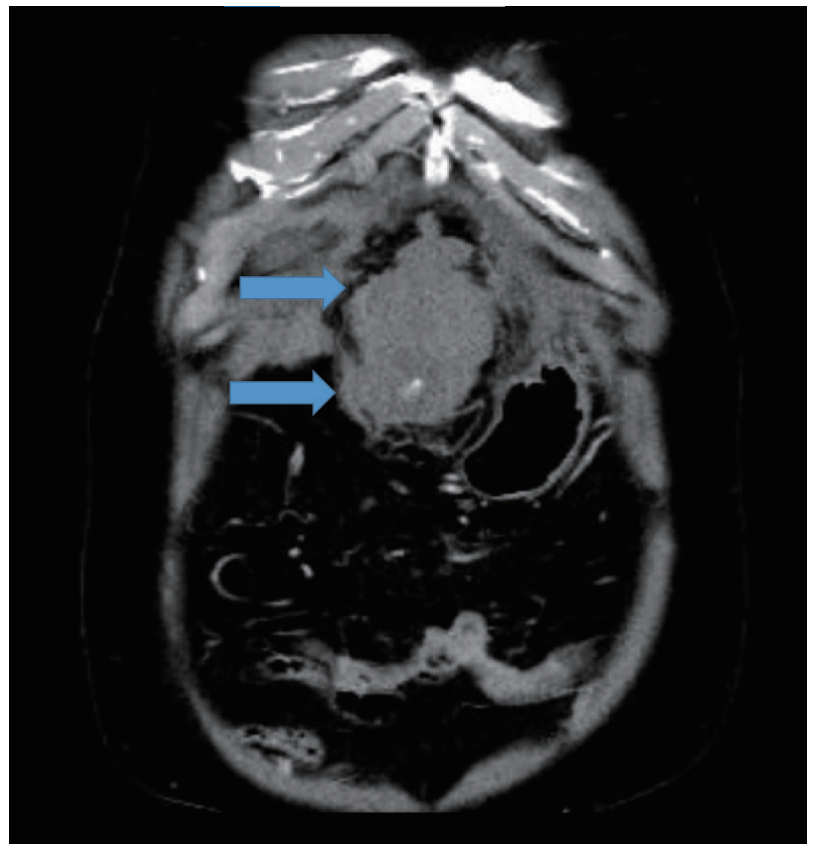

Fig. 2. The coronal image of the extraperitoneal hematoma and extravasation (blue arrows).

result in delayed complications (e.g., abdominal wall herniation with bowel obstruction or strangulation) (3).

Patients with abdominal trauma, undergoing anticoagulation therapy, should be closely monitored for abdominal wall hematoma. According to a retrospective study, 16 of the 18 abdominal wall hematoma patients who underwent angiography in a single center had altered coagulation status where 14 had drug-induced coagulopathy (1). Another retrospective study reported that six out of 10 patients who were under anticoagulants were diagnosed with RSH. In other words, the previous anticoagulation therapy was the 
most common predisposing factor. Among the remaining, two occurred after laparoscopic trocar insertion and two after a car accident and fall from a horse (4), which means that direct injury of the epigastric arteries of the rectus muscle resulted in RSH (5).

Conservative treatments (e.g., rest, analgesia, hematoma compression, anticoagulation reversal, and blood transfusion) if needed are sufficient in most abdominal wall hematoma cases. However, angiographic embolization or emergent surgery should be performed if the patient is hemodynamically unstable without a response to fluid resuscitation, shows enlarging hematoma, or if abdominal compartment syndrome is suspected (2). Empiric embolization can be performed even if the patient is stable if extravasation or pseudoaneurysm is observed on CT (1). Ultrasonography is an inexpensive, safe, and easy-to-use diagnostic method and shows a sensitivity of $80 \%-90 \%$ (2). So it may be useful to follow-up without repetitive CT imaging to determine a plan. Conservative management could be feasible without repeated imaging or invasive treatment in this case because the patient was hemodynamically stable throughout the hospital course and physical examination revealed no significant change.

In conclusion, physical examination, hemodynamic monitoring, and laboratory tests may be the important factors in determining the therapeutic plan of the abdominal wall hematoma, rather than follow-up images. Angiographic embolization or surgical management should be considered if those factors deteriorate. Patients with abdominal trauma who have coagulopathy or are under anticoagulation should carefully be monitored for abdominal wall hematoma. Detecting abdominal wall hematoma is important because it may be the clue of potential intra-abdominal organ injury or delayed complication. Future studies are needed for traumatic umbilical vessel injury.

\section{Conflict of interest}

No potential conflict of interest relevant to this article was reported.

\section{References}

1. Tung K, Naidu S, Knuttinen M, Kriegshauser J. Interventional management of anterior abdominal wall hematomas: single center experience. J Vasc Int Rad. 2016;27(3):S149.

2. Hatjipetrou A, Anyfantakis D, Kastanakis M. Rectus sheath hematoma: a review of the literature. Int J Surg. 2015; 13:267-71.

3. Matalon SA, Askari R, Gates JD, Patel K, Sodickson AD, Khurana B. Don't forget the abdominal wall: imaging spectrum of abdominal wall injuries after nonpenetrating trauma. Radiographics. 2017;37(4):121835.

4. Salemis NS, Gourgiotis S, Karalis G. Diagnostic evaluation and management of patients with rectus sheath hematoma. A retrospective study. Int J Surg. 2010;8(4):290-3.

5. Kasotakis G. Retroperitoneal and rectus sheath hematomas. Surg Clin North Am. 2014;94(1):71-6. 